\title{
Ischemic maculopathy in zidovudine-induced anemia in an HIV-positive man
}

\author{
Kathir Yoganathan' \\ Michael Austin ${ }^{2}$ \\ 'Department of Genito-Urinary \\ Medicine, ${ }^{2}$ Department of \\ Ophthalmology, Singleton Hospital, \\ Sketty, Swansea, SA2 8QA, UK
}

\begin{abstract}
Opportunistic cytomegalovirus (CMV) infection is common in severely immunocompromised HIV-positive patients. The retina is the commonest site of involvement with hemorrhages a prominent feature. CMV retinitis affects $40 \%$ of HIV positive patients who have CD4 count of less than $100 \mathrm{cell} / \mathrm{mm}^{3}$, and in these circumstances clinicians are likely to consider commencing anti-CMV therapy without considering other causes. We report a man with HIV who developed bilateral retinal hemorrhages and ischemic maculopathy in association with zidovudine (AZT)-induced anemia. Retinal hemorrhages resolved following blood transfusion.
\end{abstract}

Keywords: Ischemic maculopathy, retinal hemorrhages, anemia, zidovudine, HIV

Opportunistic cytomegalovirus (CMV) infection is common in severely immunocompromised HIV-positive patients. The retina is the commonest site of involvement with hemorrhages a prominent feature (Sarraf and Ernest 1996; Vrabec 2004). CMV retinitis affects $40 \%$ of HIV positive patients who have CD4 count of less than $100 \mathrm{cell} / \mathrm{mm}^{3}$, and in these circumstances clinicians are likely to consider commencing anti-CMV therapy without considering other causes (Cunningham and Margolis 1998). We report a man with HIV who developed bilateral retinal hemorrhages and ischemic maculopathy in association with zidovudine (AZT)-induced anemia.

\section{Case report}

A 41-year-old Caucasian homosexual man, HIV-positive since 1992, presented with oral hairy leucoplakia. Hematological indices were; CD4 113 cells $/ \mathrm{mm}^{3}$, hemoglobin (Hb) $13.3 \mathrm{~g} / \mathrm{dl}$, WBC $3.8 \times 10^{9} / 1$, and platelets $91 \times 109 / 1$. HIV RNA level was 35,000 copies/ $\mathrm{ml}$. The combination of zidovudine, lamivudine, and efavirenz was commenced.

Having defaulted, he re-presented ten weeks later with acute shortness of breath and lethargy. Examination revealed pallor but no purpura, with $\mathrm{Hb} 3.2$, WBC 1.7, and platelets 54. Serum B12, folate, and ferritin were normal. Syphilis and Parvovirus antibodies were negative. He also complained of a sub-acute painless decrease in vision affecting his right eye. Visual acuities were 6/60 right and 6/6 left. Fundoscopy revealed bilateral signs of retinal ischemia with deep "blot" retinal haemorrhages, cotton wool spots and a foveal premacular hemorrhage (Figure 1). Ophthalmic findings were otherwise normal.

Following transfusion, hemoglobin was 11.2, WBC 1.9, and platelets 40. CMV PCR was negative for a venous blood sample.

His anemia was attributed to zidovudine which was stopped. Improvement of visual acuity and retinal features ensued (Figure 2). One year later visual acuities were 6/6 right and left with significantly improved normal retinal appearances. 


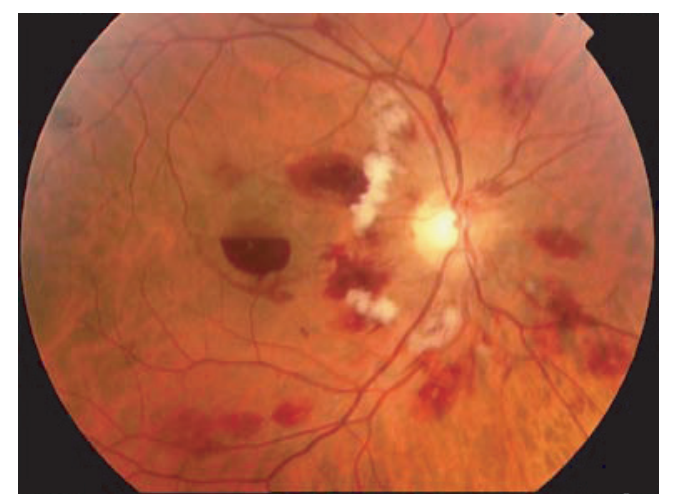

Figure I Right ocular fundus showing signs of retinal ischemia with venous dilatation, cotton wool spots, intra and preretinal hemorrhages.

\section{Discussion}

CMV retinitis may cause blindness and prompt therapy is indicated. Presenting features include painless visual deterioration, visual field defects, retinal hemorrhages, and associated pale areas of opaque retina (Cunningham and Margolis 1998; Vrabec 2004). Other typical features of CMV retinitis which may help in differential diagnosis include an initially unilateral presentation, starting in the retinal periphery with areas of hemorrhage and adjacent infiltrate giving rise to the so-called "cheese pizza" appearance. Lesions tend to increase in extent in a contiguous fashion ("brush-fire" spread). As more posterior retina becomes involved, atrophy and scarring develop in the previously inflamed areas. The vitreous is disproportionately uninflamed compared with the severity of the chorioretinal disease.

Retinal findings may be decisive in the initiation of antiCMV therapy. But other conditions may also present with similar signs. Hemorrhagic retinopathy has been described with retinal venous occlusion in HIV-positive patients

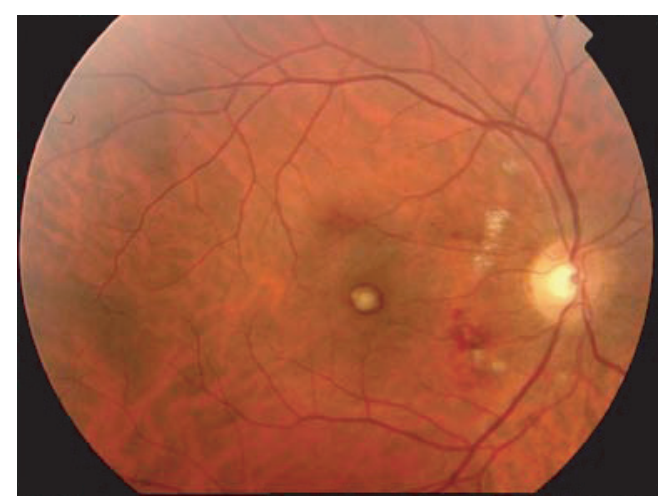

Figure 2 Right fundus 3 weeks following treatment for anemia, which shows significant improvement in features shown in Figure I.
(Friedman and Margo 1995; Mansour et al 1996). Retinal hemorrhages as part of anemia associated ischemic retinopathy have been reported in myeloproliferative disorder (Blood et al 1997) and pernicious anemia (Gupta et al 2001). These patients were HIV-negative. Beare and colleagues (2002) report two cases similar to ours in their survey of ocular disease in patients with tuberculosis and HIV presenting with fever in Africa. These individuals had hemoglobin of less than $4.0 \mathrm{~g} / \mathrm{dl}$ and widespread retinal hemorrhages including many which were white-centred, as exhibited in our patient (Figure 1).

Ischemic maculopathy in HIV is recognized but uncommon, and in most of such cases associated with CMV retinitis (Cunningham et al 2001). One of the serious adverse effects of AZT is anemia due to bone marrow suppression. It usually occurs within six weeks of initiation of therapy (Fischl et al 1990). The only risk factor identified for anemia in our patient was AZT therapy. Features of acute retinal ischemia include venous dilatation and beading, the presence of numerous retinal hemorrhages and cotton wool spots (CWS). CWS in microangiopathic HIV retinopathy tend to be small - our patient's CWS were large, possibly reflecting a greater degree of retinal ischemia (Mansour et al 1990).

Our patient showed no evidence of any other condition associated with retinal hemorrhages or ischemia. As in diabetic retinopathy, the vascular and other features of ischaemia may reverse following management - in our case by blood transfusion.

Clinicians should be aware of other causes of retinal hemorrhages in HIV-positive patients. Careful clinical examination and ophthalmic review facilitate appropriate management, avoiding costly and toxic anti-CMV treatment. Patients commencing antiretroviral therapy should be counseled regarding the importance of close follow-up and supervision.

\section{References}

Beare NAV, Kublin JG, Lewis DK, et al. 2002. Ocular disease in patients with tuberculosis and HIV presenting with fever in Africa. $\mathrm{Br} J$ Ophthalmol, 86:1076-79.

Blood AM, Lowenthal EA, Nowakowski RW. 1997. Retinopathy secondary to anemia from myeloid metaplasia in polycythemia vera. J Am Optom Assoc, 68:734-8.

Cunningham ET Jr, Levinson RD, Jampol LM, et al. 2001. Ischemic maculopathy in patients with acquired immunodeficiency syndrome. Am J Ophthalmol, 132:727-33.

Cunningham ET Jr, Margolis TP. 1998. Ocular manifestations of HIV infection. $N$ Engl J Med, 339:236-44.

Fischl MA, Parker CB, Pettinelli C, et al. 1990. The AIDS Clinical Trials Group. A randomized controlled trial of a reduced dose of zidovudine in patients with the acquired immunodeficiency syndrome. $N$ Engl $J$ Med, 323:1015-21. 
Friedman SM, Margo CE. 1995. Bilateral central retinal vein occlusions in a patient with acquired immunodeficiency syndrome. Arch of Ophthalmol, 113:1184-8.

Gupta V, Bremner FD, Telfer P. 2001. Bilateral retinal haemorrhages: an unusual presentation of pernicious anaemia. Br J Haematol, 112:831.

Mansour AM, Li H, Segal EI. 1996. Picture resembling hemicentral retinal vein occlusion in the acquired immunodeficiency syndrome: is it related to cytomegalovirus? Ophthalmologica, 210:108-11.

Mansour AM, Rodenko G, Dutt R. 1990. Half-life of cotton-wool spots in the acquired immunodeficiency syndrome. Int JSTD AIDS, 1:132-3.

Sarraf D, Ernest JT. 1996. AIDS and the Eyes. Lancet, 348:525-28

Vrabec TR. 2004. Posterior segment manifestations of HIV/AIDS. Surv Ophthalmol, 49:131-57. 
